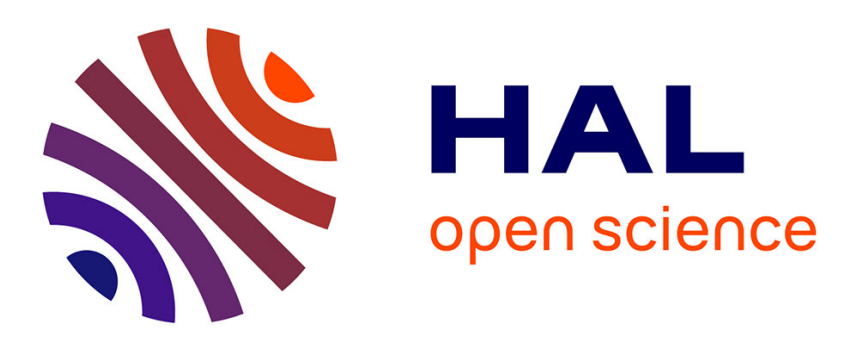

\title{
Reciprocal and advanced generation hybrids between Corymbia citriodora and C. torelliana: forestry breeding and the risk of gene flow
}

Geoffrey Dickinson, Helen Wallace, David Lee

\section{- To cite this version:}

Geoffrey Dickinson, Helen Wallace, David Lee. Reciprocal and advanced generation hybrids between Corymbia citriodora and C. torelliana: forestry breeding and the risk of gene flow. Annals of Forest Science, 2012, 70 (1), pp.1-10. 10.1007/s13595-012-0231-2 . hal-01201452

\section{HAL Id: hal-01201452 \\ https://hal.science/hal-01201452}

Submitted on 17 Sep 2015

HAL is a multi-disciplinary open access archive for the deposit and dissemination of scientific research documents, whether they are published or not. The documents may come from teaching and research institutions in France or abroad, or from public or private research centers.
L'archive ouverte pluridisciplinaire HAL, est destinée au dépôt et à la diffusion de documents scientifiques de niveau recherche, publiés ou non, émanant des établissements d'enseignement et de recherche français ou étrangers, des laboratoires publics ou privés. 


\title{
Reciprocal and advanced generation hybrids between Corymbia citriodora and $C$. torelliana: forestry breeding and the risk of gene flow
}

\author{
Geoffrey R. Dickinson • Helen M. Wallace • David J. Lee
}

Received: 16 April 2012 / Accepted: 27 July 2012 /Published online: 17 August 2012

(C) INRA / Springer-Verlag France 2012

\begin{abstract}
- Context Corymbia F1 hybrids have high potential for plantation forestry; however, little is known of their reproductive biology and potential for genetic pollution of native Corymbia populations.

- Aims This study aims to quantify the influence of reproductive isolating barriers on the success of novel reciprocal and advanced generation Corymbia hybrids.

- Methods Two maternal taxa, Corymbia citriodora subsp. citriodora and Corymbia torelliana, were pollinated using five paternal taxa, C. citriodora subsp. citriodora, C. torelliana, one C. torelliana $\times$ C. citriodora subsp. citriodora hybrid and two $C$. torelliana $\times$ C. citriodora subsp. variegata hybrids. Pollen tube, embryo and seed development were assessed.

- Results Reciprocal hybridisation between C. citriodora subsp. citriodora and C. torelliana was successful. Advanced generation hybrids were also created when $C$. citriodora subsp. citriodora or C. torelliana females were backcrossed with F1 hybrid taxa. Prezygotic reproductive isolation was identified via reduced pollen tube numbers in
\end{abstract}

\section{Handling Editor: Bruno Fady}

Contribution of the co-authors Helen M. Wallace: Primary supervisor of the $\mathrm{PhD}$ project this work was included in.

David J. Lee: Supervisor of the $\mathrm{PhD}$ project this work was included in.

G. R. Dickinson

Department of Agriculture Fisheries and Forestry-Queensland, P.O. Box 1054, Mareeba, QLD 4880, Australia

G. R. Dickinson $(\bowtie) \cdot H$. M. Wallace $\cdot$ D. J. Lee

University of the Sunshine Coast,

Maroochydore DC, QLD 4558, Australia

e-mail: geoff.dickinson@daff.qld.gov.au

D. J. Lee

Department of Agriculture Fisheries and Forestry-Queensland, P.O. Box 395, Gympie, QLD 4570, Australia the style and reduced numbers of ovules penetrated by pollen tubes. Reproductive isolation was weakest within the C. citriodora subsp. citriodora maternal taxon, with two hybrid backcrosses producing equivalent capsule and seed yields to the intraspecific cross.

- Conclusions High hybridising potential was identified between all Corymbia species and F1 taxa studied. This provides opportunities for advanced generation hybrid breeding, allowing desirable traits to be amplified. It also indicates risks of gene flow between plantation and native Corymbia populations.

Keywords Corymbia $\cdot$ Hybrid · Backcross · Isolation · Genetic pollution $\cdot$ Gene flow

\section{Introduction}

Corymbia (Myrtaceae), comprising over 113 tree species, is a sister genus to Eucalyptus and is a major component of tropical and subtropical woodland and forest floras across northern Australia (Hill and Johnson 1995). Natural forests dominated by spotted gum species (section Maculatae) occur widely throughout eastern Australia from high rainfall areas on coastal ranges through to drier inland sites (CPBR 2006). Spotted gums are commercially very important and are amongst the most commonly harvested native hardwood timbers in the Australian states of Queensland and New South Wales (Lewis et al 2010). Many eucalypt species (Corymbia and Eucalyptus) are of global economic significance with $>19$ Mha established as timber plantations (Iglesias-Trabado and Wilstermann 2008). Corymbia species and their F1 hybrids are of increasing importance to eucalypt plantation forestry due to their wide adaptability to marginal tropical and subtropical environments and high 
quality, durable timber (Lee 2007). Over 20,000 ha of Corymbia plantations has been established across Australia since the late 1990s (Barbour et al. 2008), primarily using Corymbia citriodora subsp. variegata. Selected Corymbia hybrids offer advantages over their parental species, such as superior growth, disease, insect and frost tolerance, exhibited across a wide range of environments (Lee 2007; Lee et al. 2009; Verma and Sharma 2011). In Australia, F1 Corymbia hybrids are derived by controlled pollination using Corymbia torelliana (section Torellianae) as the maternal taxon and species from the spotted gum group (section Maculatae); C. citriodora subsp. citriodora, C. citriodora subsp. variegata or Corymbia henryi, as the paternal taxon (Lee 2007; Dickinson et al. 2010). Many of the areas in eastern Australia suitable for future Corymbia species and hybrid plantations are in close proximity to natural spotted gum populations. This raises concerns of pollen-mediated gene flow from Corymbia plantations into sympatric native populations.

Hybridisation results in gene flow between individuals, contributing to plant speciation and evolution (Potts et al. 2003; Arnold and Martin 2010; Costa e Silva et al. 2012). Hybridisation can also cause genetic pollution via the pollen-mediated movement and introgression of foreign genes from exotic populations into sympatric native populations (Schierenbeck and Ellstrand 2009; Barbour et al. 2010). Genetic pollution is an increasing global concern, threatening the genetic purity of some native Populus species in Europe (Vanden Broeck et al. 2005; Ziegenhagen et al. 2008) and Eucalyptus (Potts et al. 2003; Barbour et al. 2006, 2007, 2010) and Corymbia (Barbour et al. 2008) species in Australia. Gene introgression occurs first by hybridisation and then by repeated backcrossing to one or both parents. This results in the alteration of natural patterns of genetic diversity and can potentially threaten the extinction of pure species (Vanden Broeck et al. 2005; Barbour et al. 2010). Gene introgression is controlled by ecological and genetic factors with hybrid populations often restricted to narrow hybrid zones at native population boundaries (Vanden Broeck et al. 2005; Barbour et al. 2006, 2010). Small, fragmented native populations with low genetic diversity, often of rare and threatened species, are under the greatest threat due to their vulnerability to reproductive swamping (Ziegenhagen et al. 2008; Field et al. 2009).

Gene flow between individuals is controlled by environmental and endogenous isolating barriers which act to inhibit hybridisation and maintain species integrity (Potts and Wiltshire 1997). Endogenous reproductive isolating barriers may be structural, such as disparity in flower morphology of the parent species (Gore et al. 1990) or physiological, whereby genetic combining irregularities between the parental species interrupt or impede reproduction and development (Pound et al. 2003; Wallwork and Sedgley 2005;
Suitor et al. 2008; Dickinson et al. 2012). Reproductive isolation can continue after seeds are produced, resulting in mortality and abnormal growth at germination, during seedling development and as trees (Lopez et al. 2000; Barbour et al. 2006; Volker et al. 2008; Lee et al. 2009; Costa e Silva et al. 2012). The activity of endogenous isolating barriers decreases with increasing taxonomic affinity between parents (Griffin et al. 1988; Ellis et al. 1991). Infrageneric Corymbia clades are closely related (Hill and Johnson 1995; Parra-O et al. 2009) and have an unusually high propensity to form interspecific hybrids across taxonomic groups (Griffin et al. 1988; Dickinson et al. 2012). Hybridisation may also be greater when F1 hybrids are backcrossed with either parental species (Potts et al. 2000, 2003). A better understanding of the breeding system and reproductive biology of Corymbia species and their F1 hybrids is required to minimise future gene flow risks (Barbour et al. 2008).

Australian Corymbia hybrid programs have focused on the production of $\mathrm{F} 1$ hybrids using $C$. torelliana as the maternal parent and a limited number of spotted gum taxa and families as the paternal parents (Lee 2007, Lee et al. 2009). Interspecific F1 hybridisation between $C$. torelliana and other taxa is controlled by prezygotic isolating barriers, with reproductive success increasing with increasing taxonomic affinity of parental taxa (Dickinson et al. 2012). There has, however, been little investigation of the reproductive success of advanced generation $C$. torelliana hybrids. Knowledge of the hybridising potential of $C$. citriodora as the maternal parent is also poorly known, despite its international importance as a forestry plantation species and wide natural distribution across eastern Australia. This study aims to quantify the reproductive success of reciprocal C. citriodora subsp. citriodora and C. torelliana hybrids, and advanced generation hybrids where both parental species were backcrossed with $C$. torelliana $\times C$. citriodora subsp. citriodora or C. torelliana $\times C$. citriodora subsp. variegata $\mathrm{F} 1$ hybrid taxa. The activity and locations of reproductive isolation for these novel crosses will be determined via the assessment of pollen-pistil interactions, embryo development and seed germination. Improved knowledge of the reproductive biology of $C$. citriodora subsp. citriodora, $C$. torelliana and their F1 hybrids will benefit Corymbia hybrid breeding and help quantify potential risks of pollen-mediated gene into native Corymbia populations.

\section{Materials and methods}

\subsection{Parent details}

Three $C$. citriodora subsp. citriodora and three $C$. torelliana maternal parent trees were crossed with five paternal taxon 
treatments, C. torelliana, C. citriodora subsp. citriodora, $C$. torelliana $\times C$. citriodora subsp. citriodora hybrid $(\mathrm{CT} \times$ CCC hybrid), C. torelliana $\times C$. citriodora subsp. variegata hybrid $1(\mathrm{CT} \times \mathrm{CCV}$ hybrid 1$)$ and $C$. torelliana $\times C$. citriodora subsp. variegata hybrid $2(\mathrm{CT} \times \mathrm{CCV}$ hybrid 2$)$ during 2007-2008. All maternal parent trees were $>8 \mathrm{~m}$ tall and were selected from amenity plantings of unknown genetic origin, near Mareeba, $17.00^{\circ} \mathrm{S}, 145.43^{\circ} \mathrm{E}$, Queensland, Australia. Pollen was collected from three individuals per taxon for the $C$. torelliana and $C$. citriodora subsp. citriodora cross treatments (maternal parent trees were not used as pollen parents) and one individual per taxon for the three hybrid backcross treatments. Flowers were collected prior to opening, placed in vases in the laboratory and anthers harvested after operculum shedding. The pollen was then extracted, dried for $72 \mathrm{~h}$ in a silica-gel desiccator and stored in gel capsules at $4{ }^{\circ} \mathrm{C}$ until required. A pollen polymix was made for the $C$. torelliana and $C$. citriodora subsp. citriodora cross treatments. Pollen viability was confirmed 2 weeks prior to pollination.

\subsection{Pollinations}

Controlled pollinations were conducted using the conventional pollination method (Dickinson et al. 2010) on both maternal parent species between August and September, with flowers accessed using an 8-m elevated platform. Each cross treatment was randomly allocated to three flower bunches on each maternal parent tree. Flower bunches were selected prior to pollination, if most buds were yellow and within $0-3$ days of natural operculum lift. Open flowers and immature, overripe and excessive buds were then removed, retaining approximately $50-100$ buds per bunch. The retained flowers were emasculated using pollination pliers and covered with an exclusion bag. Flowers were pollinated approximately 7 days later when the stigmas had visible exudate. Exclusion bags were re-applied and retained for a further 7 days, then removed. Samples were collected 1 and 5 weeks after pollination by randomly harvesting three to four capsules per bunch. These were pooled to give 10 samples per cross treatment for each maternal taxon, thus giving 30 replicates per controlled cross combination for each time point sampled. All remaining capsules for each maternal taxon were harvested at maturity and assessed individually as described below.

\subsection{Sample collection and assessment}

Samples were collected 1 week after pollination for measurement of pollen tube growth and embryo fertilisation. Ten flowers per cross treatment were collected for each maternal taxon, fixed in Carnoy's solution (60\% ethyl alcohol [95\%], $30 \%$ chloroform, $10 \%$ glacial acetic acid) and stored at $4{ }^{\circ} \mathrm{C}$. Samples were rinsed in distilled water three times then softened by autoclaving at $121{ }^{\circ} \mathrm{C}$ for $20 \mathrm{~min}$ in a solution of $0.8 \mathrm{~N}-\mathrm{NaOH}$. Samples were then rinsed in distilled water and stained in a solution of decolourised aniline blue. Pistils were dissected from the developing capsule and cut longitudinally using a scalpel blade, exposing the transmitting tissue and pollen tubes for pollen tube measurement. Pistils were then squashed onto slides with the cut surface of both halves facing upright and viewed under fluorescence using a Zeiss Axioskop (2 MOT) microscope. The transmitting tissue midway along the length of the style was examined and the number of pollen tubes counted. One of the three locules in each flower was randomly selected and the ovules dissected and counted using a stereo microscope. Ovules were then mounted on a slide and viewed using fluorescent microscopy. Fertilised ovules were counted where pollen tubes were observed to have penetrated the ovule micropyle.

Ten developing capsules per cross treatment were harvested at 5 weeks after pollination, fixed in FPA50 (90\% ethyl alcohol [50\%], $5 \%$ formalin, $5 \%$ propionic acid) and stored at $4{ }^{\circ} \mathrm{C}$. Capsules were dissected and the number and size of developing embryos were assessed. Developing embryos were distinguished from other embryos by size $(>700 \mu \mathrm{m})$ and appearance, with developing embryos well hydrated and yellow/white in colour. Mature seeds and capsules were harvested and counted; 12 weeks after pollination for $C$. torelliana and 21 weeks after pollination for $C$. citriodora subsp. citriodora when the seeds were mature. Percentage capsule retention at maturity was calculated for each bunch (excluding subsets sampled at 1 and 5 weeks after pollination) and averaged for each cross treatment. Capsules were dried individually, seed extracted and the seed number per capsule measured. Seed viability was assessed for each cross treatment on each maternal tree, with 30 seeds $\times 3$ replicates sown onto germination trays. The trays were placed into a germination cabinet and incubated at $25{ }^{\circ} \mathrm{C}$ for 10 days. Germinated seed was counted and germination percent calculated.

\subsection{Statistical analysis}

All data was screened for assumptions of normality and homogeneity of variance prior to analysis. Statistical analysis was conducted using Genstat 11.1 statistical software (Copyright 2008, VSN International Ltd). Measurement parameters for each maternal taxon were analysed separately via a linear mixed model using restricted maximum likelihood to estimate variance. Model inputs included: individual measurement parameters as the response variate, pollen parent as the fixed effect and maternal parent as the random effect. Error terms were automatically calculated and added to models. Where significant differences in the Wald statistic were detected, these were tested using the post 
hoc least significant difference test $(P<0.05)$ allowing pairwise comparisons between means.

\section{Results}

\subsection{C. citriodora subsp. citriodora maternal taxon}

Our results show high fertility between all crosses at all stages of development. High numbers of pollen tubes (4571 per flower) were found within the mid stylar region of the C. citriodora subsp. citriodora females (Fig. 1a, b) for all crosses examined. The most successful paternal cross treatment was the intraspecific $C$. citriodora subsp. citriodora cross, which recorded a significantly higher $(P<0.05)$ number of pollen tubes than all crosses, except the $\mathrm{CT} \times \mathrm{CCC}$ hybrid cross (Fig. 2a). High numbers of pollen tubes were also observed to have penetrated the ovule micropyle (10.217.7 per flower) for all crosses (Fig. 1c). The highest number of penetrated ovules was observed for the intraspecific C. citriodora subsp. citriodora cross, which was significantly higher $(P<0.05)$ than all treatments, except the $\mathrm{CT} \times \mathrm{CCV}$ hybrid 2 cross (Fig. 3a). All crosses had developing embryos 5 weeks after fertilisation (Fig. 1d). The intraspecific $C$. citriodora subsp. citriodora cross had the most developing embryos, significantly more $(P<0.01)$ than all other crosses (Fig. 4a). There was no difference in developing embryo numbers between the other cross treatments. All crosses with $C$. citriodora subsp. citriodora as the maternal parent produced seed at maturity, with a similar seed number per capsule for the $C$. citriodora subsp. citriodora, $\mathrm{CT} \times$ CCC hybrid and CT $\times$ CCV hybrid 2 crosses (Fig. 5a). The C. citriodora subsp. citriodora cross had a significantly greater $(P<0.001)$ seed yield than the interspecific $C$. torelliana cross. Poorest seed number per capsule was measured for the $\mathrm{CT} \times \mathrm{CCV}$ hybrid 1 cross, which was significantly lower than all other crosses. Capsule retention at maturity was not significantly different (Table 1). Seed viability percentage (Table 1$)$ was lowest $(P<0.01)$ for the $\mathrm{CT} \times \mathrm{CCC}$ hybrid cross $(54.2 \%)$, whereas all remaining crosses were similar (72.0-83.5 \%).

\subsection{C. torelliana maternal taxon}

Pre-zygotic reproductive success for the $C$. torelliana females was initially high across all cross treatments, with
Fig. 1 Fluorescent microscope images. a Germinating pollen on stigma surface and pollen tubes in upper style, $C$. citriodora subsp. citriodora $\times$ C. torelliana, scale bar $=500 \mu \mathrm{m}$. b Pollen tubes in mid style, C. citriodora subsp. citriodora $\times$ C. torelliana, scale bar $=500 \mu \mathrm{m}$. $\mathbf{c}$ Pollen tube penetration of the ovule micropyle, C. citriodora subsp. citriodora $\times(C$. torelliana $\times C$. citriodora subsp. citriodora hybrid), scale bar $=100 \mu \mathrm{m}$. Light microscope images of developing embryos within the locule at age 5 weeks. $\mathbf{d} C$. citriodora subsp. citriodora $\times C$. citriodora subsp. citriodora, scale bar $=2,000 \mu \mathrm{m}$. e $C$. torelliana $\times$ C. torelliana, scale bar $=2,000 \mu \mathrm{m}$
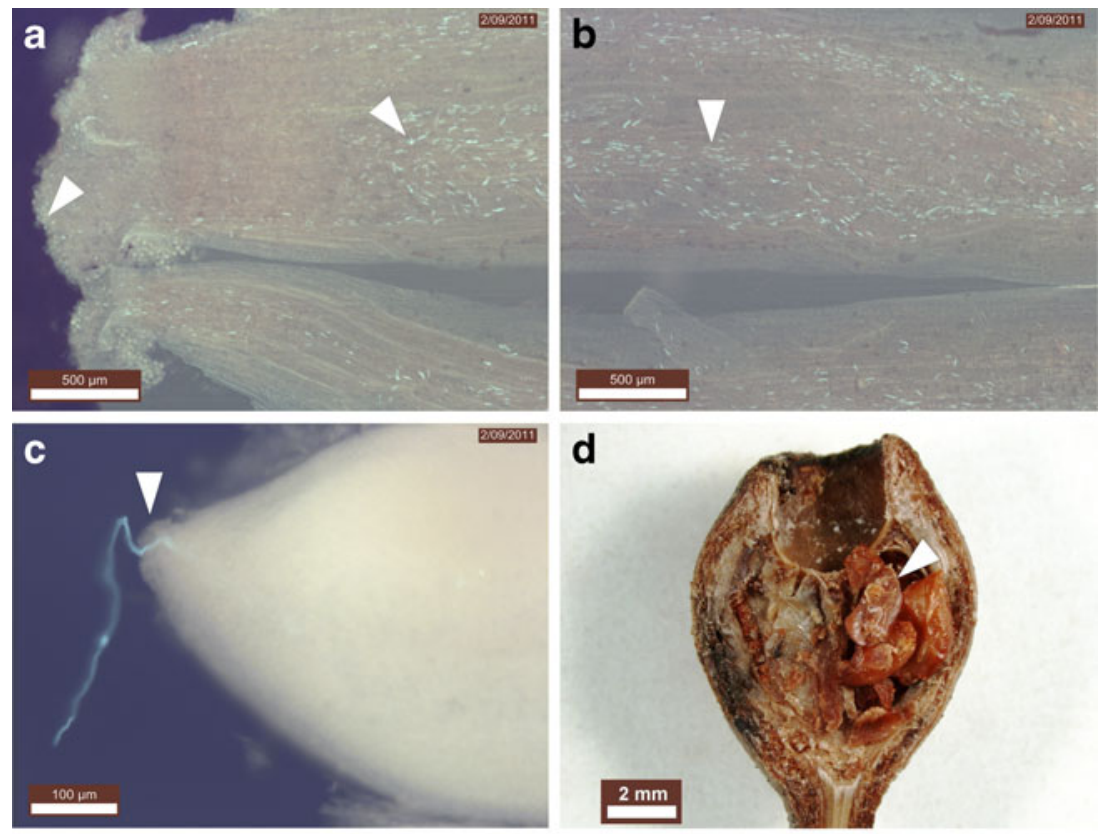

d

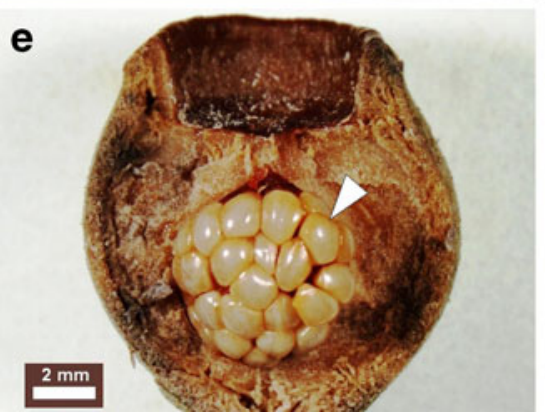

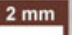



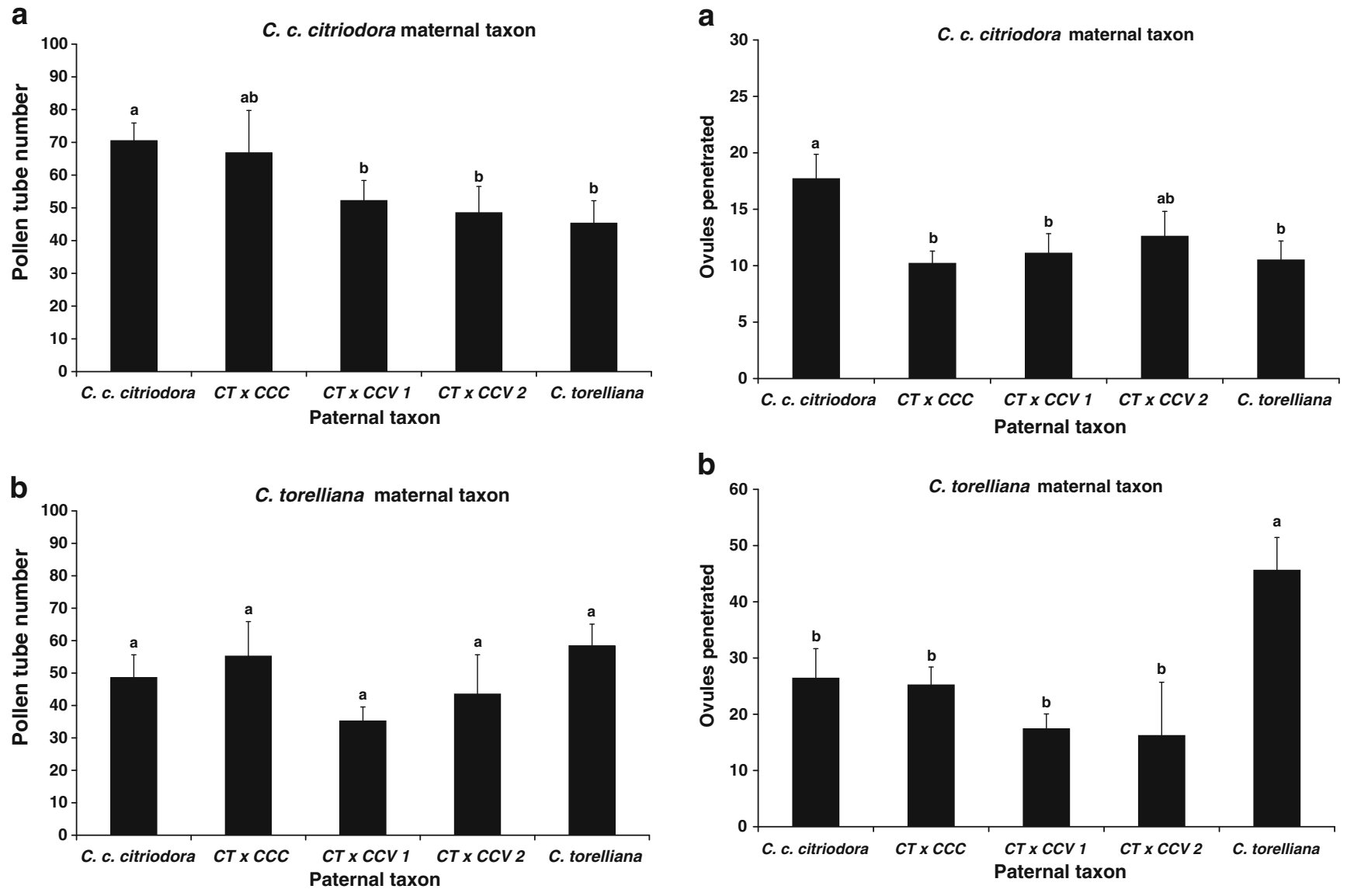

Fig. 2 Number of pollen tubes in mid-stylar region for a C. citriodora subsp. citriodora maternal taxon or b C. torelliana maternal taxon, crossed with five paternal taxa. Treatment means ( \pm s.e.) with different letters are significantly different $(P<0.05)$

similar numbers of pollen tubes observed in the middle style region (Fig. 2b). Differences between treatments were observed at the next stage of fertilisation with significantly more ovules penetrated by pollen tubes $(P<0.001)$ for the intraspecific $C$. torelliana cross compared with all other cross treatments (Fig. 3b). The number of penetrated ovules for the interspecific $C$. citriodora subsp. citriodora and the three hybrid cross treatments were similar. Five weeks after fertilisation, reproductive success remained highest for the C. torelliana cross, with the number of developing embryos (47.4 per capsule) significantly higher $(P<0.001)$ than all other crosses (Fig. 4b). The number of developing embryos per capsule also varied significantly between remaining crosses, with the interspecific $C$. citriodora subsp. citriodora cross significantly higher than the $\mathrm{CT} \times \mathrm{CCV}$ hybrid 1 and $\mathrm{CT} \times \mathrm{CCV}$ hybrid 2 crosses, but similar to the $\mathrm{CT} \times$ CCC hybrid cross. Twelve weeks after pollination, all crosses produced seed (Fig. 5b), with highest yields for the $C$. torelliana cross $(P<0.001)$. The $\mathrm{CT} \times \mathrm{CCC}$ hybrid cross had an intermediate seed yield which was significantly greater than both the $\mathrm{CT} \times \mathrm{CCV}$ hybrid 1 and $\mathrm{CT} \times \mathrm{CCV}$

Fig. 3 Number of ovules penetrated for a C. citriodora subsp. citriodora maternal taxon or $\mathbf{b} C$. torelliana maternal taxon, crossed with five paternal taxa. Treatment means $( \pm$ s.e. $)$ with different letters are significantly different $(P<0.05)$

hybrid 2 crosses. The interspecific $C$. citriodora subsp. citriodora cross was not significantly different from the three hybrid backcross treatments. Capsule retention percent at maturity was not significantly different (Table 1). Seed viability percentage (Table 1$)$ was lowest $(P<0.001)$ for the interspecific C. citriodora subsp. citriodora cross (68.3\%), whereas the intraspecific $C$. torelliana and three hybrid backcrosses were uniformly high (93.9-98.3\%).

\section{Discussion}

\subsection{Interspecific reproductive isolation}

Interspecific and advanced generation hybrids were successfully created using C. citriodora subsp. citriodora or $C$. torelliana as the maternal taxon, despite numerous prezygotic reproductive isolating barriers. Prezygotic isolation was observed early in the fertilisation process for the $C$. citriodora subsp. citriodora females, with lower numbers of pollen tubes in the style for the interspecific $C$. torelliana 

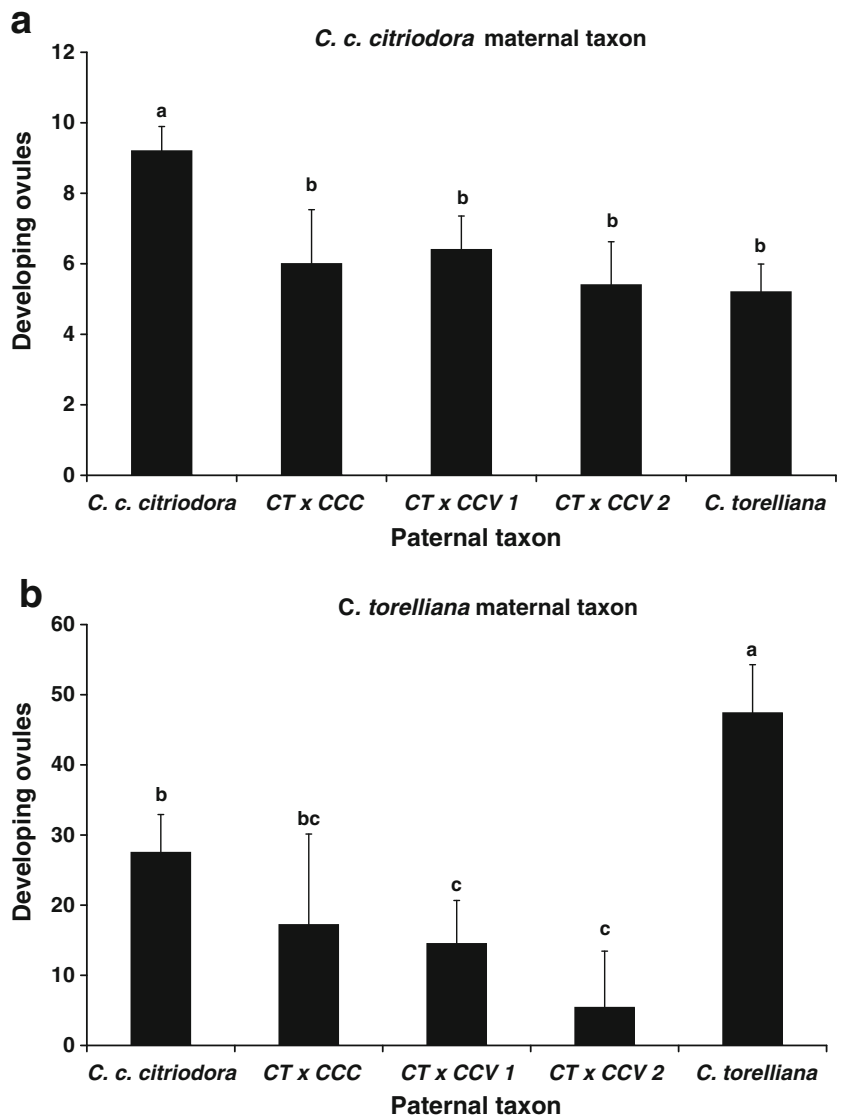

Fig. 4 Number of developing ovules 5 weeks after pollination for a $C$. citriodora subsp. citriodora maternal taxon or $\mathbf{b}$ C. torelliana maternal taxon, crossed with five paternal taxa. Treatment means ( \pm s.e.) with different letters are significantly different $(P<0.01)$

and both the CT $\times$ CCV hybrid 1 and $\mathrm{CT} \times \mathrm{CCV}$ hybrid 2 crosses. Reproductive isolation was not recorded in the $C$. torelliana females at the same stage. Early reproductive isolation in eucalypts is recognised at several prezygotic stages including impeded pollen adhesion and germination on the stigma (Dickinson et al. 2012) and disrupted pollen tube growth through the style towards the ovaries (Dickinson et al. 2012; Ellis et al. 1991; Wallwork and Sedgley 2005). The micropyle was an important site of reproductive isolation
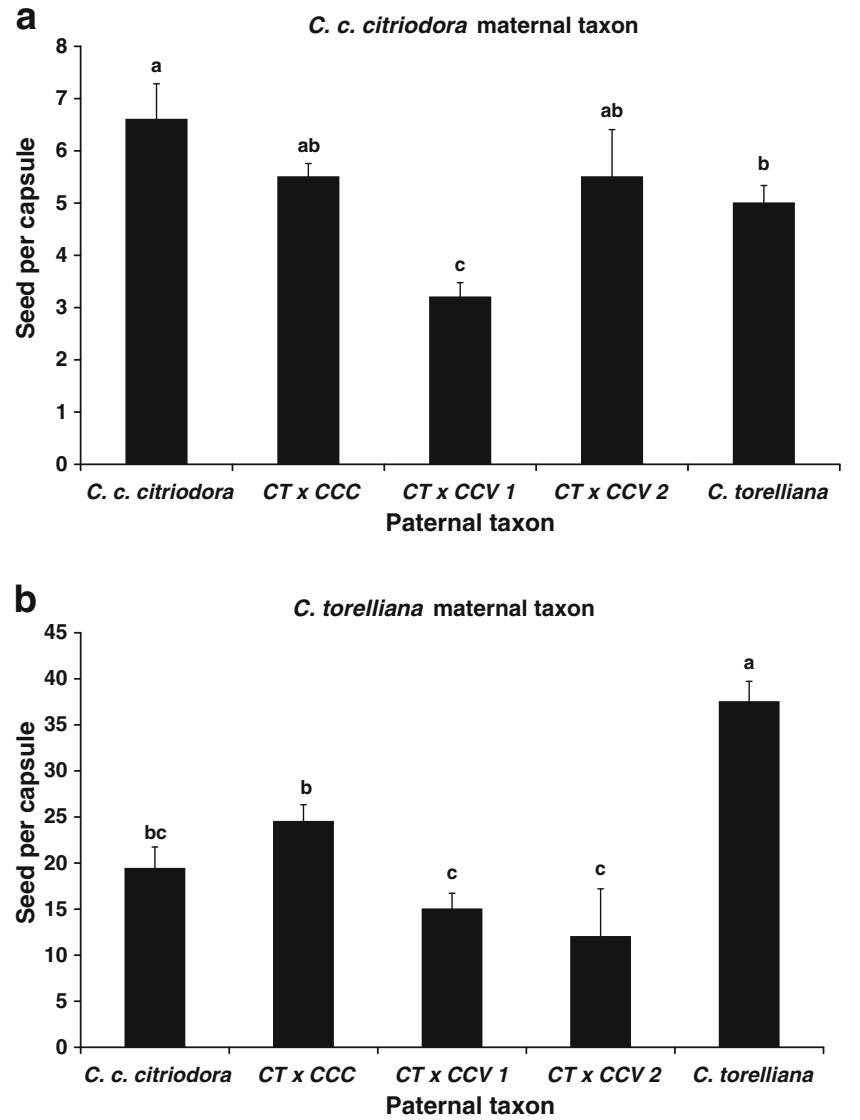

Fig. 5 Seed number per capsule for a C. citriodora subsp. citriodora maternal taxon or $\mathbf{b} C$. torelliana maternal taxon, crossed with five paternal taxa. Treatment means $( \pm$ s.e. $)$ with different letters are significantly different $(P<0.001)$

for both the C. citriodora subsp. citriodora and C. torelliana females. Almost all interspecific and advanced generation crosses had fewer ovules penetrated by pollen tubes than the intraspecific crosses. The cross between the $C$. citriodora subsp. citriodora female and the CT $\times \mathrm{CCV}$ hybrid 2 male was the only treatment with similar numbers of penetrated ovules compared with the intraspecific cross. The micropyle is one of the primary sites of interspecific reproductive isolation in Corymbia, including C. torelliana $\times$ C. citriodora subsp.
Table 1 Capsule retention percent at maturity and germination percent for C. c. citriodora or $C$. torelliana maternal taxon, crossed with five paternal taxa

Where $F$ (ddf) values were significantly different $(P<0.05)$, treatment comparisons were conducted using the LSD test. Treatment means with different letters are significantly different

\begin{tabular}{|c|c|c|c|c|}
\hline \multirow[t]{3}{*}{ Paternal taxon } & \multicolumn{4}{|l|}{ Maternal taxon } \\
\hline & \multicolumn{2}{|c|}{ C. citriodora subsp. citriodora } & \multicolumn{2}{|l|}{ C. torelliana } \\
\hline & Capsule retention $\%$ & Germination \% & Capsule retention $\%$ & Germination $\%$ \\
\hline C. citriodora subsp. citriodora & 30.3 & $72.0 \mathrm{a}$ & 11.3 & $68.3 \mathrm{~b}$ \\
\hline $\mathrm{CT} \times \mathrm{CCC}$ hybrid & 34.0 & $54.2 \mathrm{~b}$ & 22.6 & 93.9 a \\
\hline $\mathrm{CT} \times \mathrm{CCV}$ hybrid 1 & 23.4 & $72.3 \mathrm{a}$ & 17.0 & $96.1 \mathrm{a}$ \\
\hline $\mathrm{CT} \times \mathrm{CCV}$ hybrid 2 & 33.4 & $83.5 \mathrm{a}$ & 12.0 & - \\
\hline C. torelliana & 31.5 & 78.9 a & 17.8 & $98.3 \mathrm{a}$ \\
\hline$P$ value & 0.746 & 0.001 & 0.329 & $<0.001$ \\
\hline
\end{tabular}


citriodora (Dickinson et al. 2012) and is a common site of reproductive isolation in other eucalypt species (Ellis et al. 1991; Wallwork and Sedgley 2005). Reproductive isolation at prezygotic, rather then postzygotic stages increases reproductive efficiency, conserving ovules for fertilisation by more desirable pollen and minimising wastage of plant resources via embryo abortion.

Postzygotic embryo development and survival varied between the C. citriodora subsp. citriodora and C. torelliana females. Early differences in prezygotic reproductive success were carried through to seed maturity for the $C$. torelliana females indicating little activity of postzygotic isolation. The C. citriodora subsp. citriodora females, however, experienced substantial decreases in embryo numbers between fertilisation and seed maturity. Resource allocation is a major cause of postzygotic embryo degeneration and abortion in eucalypts (Pound et al. 2003; Suitor et al. 2008) and is likely the main cause of postzygotic abortion within the $C$. citriodora subsp. citriodora females. The relatively poor postzygotic reproductive success of the $\mathrm{CT} \times \mathrm{CCV} 1$ hybrid cross may also suggest some activity of postzygotic isolation. Percentage capsule retention was not influenced by cross treatment for either the C. citriodora subsp. citriodora or C. torelliana females. Capsule retention in eucalypts is controlled by fertilisation level and resource competition, which justifies the commitment of valuable resources to fruit and seed production (Suitor et al. 2008). Although seed number per capsule varied between cross treatments in our study, sufficient embryo fertilisation was achieved to ensure a similar level of capsule retention for all crosses.

Our results show low germination for seeds resulting from the $C$. citriodora subsp. citriodora female crossed with the $\mathrm{CT} \times \mathrm{CCC}$ hybrid and the $C$. torelliana female crossed with C. citriodora subsp. citriodora. Inviability in hybrid progeny is often caused by the deleterious interactions of genes from the same or different loci from the parental species and is recognised as a major impediment to progress in hybrid breeding programs (Potts and Dungey 2004; Costa e Silva et al. 2012). Inviability is expressed by high mortality and as abnormal phenotypes at germination, seedling development or as young trees (Lopez et al. 2000; Barbour et al. 2006). Reduced hybrid seed viability of Corymbia hybrids has also been identified for a range of interspecific crosses (Dickinson et al. 2012), and F1 and F2 families (Shepherd et al. 2007), when compared to intraspecific controls.

\subsection{Reciprocal hybridisation}

Reciprocal interspecific hybridisation between $C$. citriodora subsp. citriodora and C. torelliana was achieved utilising either species as the maternal or paternal taxon. Bilateral reproductive isolation is primarily caused by structural differences in flower morphology between parent species, particularly disparity in flower size (Gore et al. 1990). C. torelliana and C. citriodora subsp. citriodora have relatively similar flower size (CPBR 2006). Interspecific reproductive productivity was, however, much lower for the $C$. citriodora subsp. citriodora females (5.0 seeds per capsule) compared to the C. torelliana females (19.4 seeds per capsule). Spotted gums are recognised for their much lower seed numbers per capsule than C. torelliana, with Lee (2007) suggesting hybrid seed yields can be up to four times higher when $C$. torelliana is used as the maternal taxon, rather than a spotted gum species. The higher seed productivity of $C$. torelliana and a propensity for more regular and prolific flowering are primary reasons why large-scale Corymbia hybrid controlled-cross pollination programs in Australia are conducted using $C$. torelliana exclusively as the maternal taxon (Lee 2007; Dickinson et al. 2010).

The ability to hybridise reciprocally between $C$. citriodora subsp. citriodora and $C$. torelliana may provide opportunities for the development of new genetic combinations with desirable phenotypes. The direction of the cross can influence the heritability of traits within hybrid progeny (Assis 2000; Barbour et al. 2007; Rix et al. 2012) and the genetic interactions which affect reproductive isolation and hybrid inviability (Potts and Dungey 2004). Corymbia F1 hybrids have partial dominance towards the $C$. torelliana parent for the traits of increased rooting of cuttings (Assis 2000), frost resistance and Quambalaria pitereka disease tolerance (Lee et al. 2009) and towards the spotted gum parent for branching and straightness (Lee et al. 2009). C. citriodora subsp. citriodora and C. citriodora subsp. variegata have some desirable morphological characteristics which are superior to $C$. torelliana, including wider environmental adaptability (rainfall, 600-2,000 mm/year), more desirable tree architecture (greater straightness, less branching) and better wood quality (CPBR 2006; Lee 2007). A reciprocal cross utilising a spotted gum species as the maternal taxon, may result in progeny with desirable wood traits more closely associated with spotted gum, which can be screened for plantation forestry potential.

\subsection{Advanced generation hybridisation}

Advanced generation backcross Corymbia hybrids were created with both the $C$. citriodora subsp. citriodora and C. torelliana maternal taxon, when backcrossed with the $C$. torelliana $\times$ C. citriodora subsp. citriodora hybrid and two C. torelliana $\times$ C. citriodora subsp. variegata hybrid paternal taxa. To date, only limited research has been conducted on advanced generation Corymbia hybrids; with variable survival and seedling performance of controlled-cross pollinated F2 families (Shepherd et al. 2007) or spontaneous F2 and F3 families (Verma and Sharma 2011). Advanced 
generation eucalypt hybrids including F2's, backcrosses and three-way crosses are often disposed to hybrid breakdown due to disruption of co-adapted gene complexes, loss or duplication of chromosomal segments and unfavourable epistasis (Potts and Dungey 2004; Costa e Silva et al. 2012). In our study, variation in reproductive success was identified between advanced generation hybrid crosses within both maternal taxa. Variation in reproductive success between hybrid pollen families may be due to specific genetic combining interactions between individuals, known as specific hybridising ability (SHA; Nikles and Newton 1991). SHA has resulted in variable reproductive success and field performance between F1 hybrid families of $C$. torelliana (Lee et al. 2009).

Advanced generation hybrid breeding is a strategy used within hybrid tree improvement programs including Pinus (Brawner et al. 2005) and Populus (Stanton et al. 2010). The ability to create backcross advanced generation Corymbia hybrids offers new opportunities for commercial Corymbia hybrid breeding programs. Individual F1 Corymbia hybrid families have shown great promise (Lee et al. 2009); however, there would be many desirable traits more closely associated with individual parental species, which could be amplified by hybrid backcrossing. Improving the amenability of Corymbia hybrid clones to vegetative propagation is recognised as a key priority for successful hybrid commercialisation (Lee et al. 2009; Hung and Trueman 2011). C. torelliana has higher amenability to vegetative propagation than $C$. citriodora subsp. citriodora with the F1 hybrid progeny of these parental species inheriting this trait with a bias towards the C. torelliana taxon (Assis 2000). A backcross between $C$. torelliana and an F1 hybrid pollen parent could produce advanced generation hybrid progeny with greater amenability to vegetative propagation and clonal deployment. Other desirable traits of $C$. torelliana which could be amplified by backcrossing include disease tolerance to $Q$. pitereka and browsing resistance to some insect pests (Lee 2007). Alternatively, the spotted gums have greater structural timber properties including density, hardness and strength and tree form and branching characteristics than C. torelliana (Lee 2007). A backcross between $C$. citriodora subsp. citriodora and an F1 hybrid parent could produce advanced generation hybrid progeny with greater wood quality.

The Corymbia hybrid breeding program initiated in Australia in 1999 utilises a Reciprocal Recurrent SelectionSelecting Forwards strategy. Parent species are improved via recurrent selection in subpopulations, combined with concurrent screening of a wide range of $\mathrm{F} 1$ hybrid progeny combinations (Lee et al. 2009). The development of a synthetic breed using advanced generation hybrid breeding is an alternative strategy (Brawner et al. 2005; Lee et al. 2009). Advanced generation Corymbia hybrid breeding is still in its early stages and there are concerns of poor seed yields, inviability and segregation, particularly in F2 families (Shepherd et al. 2007). Advanced generation hybrid breeding has proven a successful strategy within Pinus hybrid breeding programs (Brawner et al. 2005); whereas in Populus hybrid breeding programs, their use is limited to ventures which compliment the mainstream RRS F1 strategy (Stanton et al. 2010). The results from our study suggest that advanced generation backcross hybrids and the potential development of a synthetic breed for the Corymbia complex are possible future directions for Corymbia hybrid breeding.

\subsection{Gene flow risks}

Successful hybridisation and gene flow is controlled by environmental pre-mating barriers (geographic and ecological isolation), endogenous post-mating barriers (pre- and postzygotic isolation) and interacting post-mating barriers (hybrid fitness; Potts et al. 2003). Native spotted gum populations occur throughout eastern Australia (CPBR 2006) in areas where Corymbia plantation expansion is most likely (Lee et al. 2010) and are at greater risk of genetic pollution. Native $C$. torelliana populations are at a lower risk of genetic pollution being restricted to far north Queensland (CPBR 2006) well outside the areas anticipated for Corymbia plantation development. Similarities in the reproductive ecology of $C$. citriodora, C. torelliana and their hybrids suggest some pollen flow could occur between sympatric plantation and native populations. Flowering times for native $C$. citriodora and $C$. torelliana populations are partially synchronous (CPBR 2006). Cultivated $C$. citriodora, $C$. torelliana and their F1, F2 and F3 hybrids have also exhibited some flowering synchrony (Verma et al. 1999). C. citriodora and $C$. torelliana flowers are also attractive to a wide range of generalist pollinators including bats and birds, which may facilitate pollen movement over long distances between flowering populations (Barbour et al. 2008).

Infrageneric clades within the Corymbia are closely related, which is reflected in their unusually high propensity to form hybrids across taxonomic groups (Griffin et al. 1988; Dickinson et al. 2012). C. torelliana (section Torellianae) and spotted gum species (section Maculatae) are classified within subgenus Blakella and are closely related (Parra-O et al. 2009). Our study shows that pre- and postzygotic endogenous isolating barriers between crosses of $C$. torelliana, $C$. citriodora subsp. citriodora or their hybrids are relatively weak and successful hybridisation is likely if environmental pre-mating barriers are ineffective.

Inviability of F1 and advanced generation hybrids is an expression of outbreeding depression and is primarily caused by unfavourable additive $\times$ additive epistatic effects which increase with plant age (Costa e Silva et al. 2012). In our study, hybrid viability was assessed at the germination 
stage, with the seed germination percentage of hybrid crosses generally equivalent to intraspecific crosses. The only cases of reduced hybrid viability were the F1 cross between $C$. torelliana and $C$. citriodora subsp. citriodora and the backcross between $C$. citriodora subsp. citriodora and the $\mathrm{CT} \times \mathrm{CCC}$ hybrid. Hybrid inviability at germination has been reported for interspecific F1 (Dickinson et al. 2012) and advanced generation (Shepherd et al. 2007) Corymbia hybrids. In many cases, however, germination of eucalypt hybrid seed is as successful as intraspecific seed (Ellis et al. 1991; Lopez et al. 2000). Hybrid inviability expressed by high mortality and as abnormal phenotypes is often most pronounced after germination during seedling development or as trees (Potts and Dungey 2004; Volker et al. 2008; Costa e Silva et al. 2012). F1 hybrid progeny typically display poor average survival and growth when compared to outcrossed parental controls; however, a small proportion of F1 hybrid families can exceed the parental performance when grown under optimum conditions (Potts and Dungey 2004). Lee et al. (2009) describes high variability in survival and growth between F1 Corymbia hybrid families to age 3 years. Hybrid inviability also varies widely between advanced generation hybrid combinations, where complex genomic interactions can result in considerable variation in hybrid survival and fitness (Potts et al. 2003; Costa e Silva et al. 2012). Eucalyptus backcross hybrids have demonstrated growth and survival rates which are superior to F1 and F2 hybrids and similar to parental species (Potts et al. 2000, 2003). The reproductive success and viability of Populus backcross hybrids, however, are often poorer than F1 hybrids (Vanden Broeck et al. 2005).

The survival chances of exotic hybrid progeny regenerating within native habitats are expected to be lower than within plantations due to harsh environmental conditions, lack of adaptation to local environments and genetic incompatibilities (Lopez et al. 2000; Barbour et al. 2006, 2010). Barbour et al. (2006) identified evidence of greater early-age selection against exotic Eucalyptus ovata $\times$ Eucalyptus nitens $\mathrm{F} 1$ hybrids in native habitats as compared to the same hybrids in a cultivated environment. The survival chance of Corymbia hybrids in native habitats is unknown. There have been numerous observations of spontaneous $C$. torelliana $\times$ spotted gum hybrids surviving within cultivated plantings (Lee 2007; Verma and Sharma 2011) however observations of spontaneous hybrids in native Corymbia populations are rare (Hill and Johnson 1995).

\section{Conclusions}

Successful hybridisation was achieved for all interspecific and advanced generation Corymbia hybrid crosses investigated in this study. These results were obtained using a limited number of maternal and paternal parents; however, they provide valuable directions for future Corymbia hybrid breeding and the management of gene flow risk. The creation of advanced generation Corymbia hybrids has breeding advantages as it will allow for the amplification of numerous desirable traits by breeding beyond the F1 hybrid generation, and may potentially allow the development of a synthetic breed for the Corymbia complex. The high hybridising propensity of these Corymbia taxa, however, suggests a risk of exotic gene flow from plantations of Corymbia species and their F1 hybrids into sympatric native Corymbia populations. Gene flow from F1 Corymbia hybrid plantations into native spotted gum forests poses the greatest risk, although the long-term viability of hybrids within native habitats is unknown. Investigation of Corymbia hybrid seed sowing and seedling trials in native forest areas to quantify longer-term hybrid viability and the risk of gene introgression is recommended.

Acknowledgments We thank Nick Kelly, Brooke Dwan and Bruce Randall for technical assistance with field pollinations and laboratory studies.

Funding This work was supported by the Department of Agriculture, Fisheries and Forestry - Queensland via the 'Smart Forests Alliance Queensland' project funded under the Smart State Fund and the 'Characterising wood properties for deployment of elite subtropical and tropical hardwoods' project funded through the Plantation Hardwood Research Fund.

\section{References}

Arnold ML, Martin NH (2010) Hybrid fitness across time and habitats. Trends Ecol Evol 25:530-536

Assis TF (2000) Production and use of Eucalyptus hybrids for industrial purposes. In: Dungey HS, Dieters MJ, Nikles DG (Eds) Hybrid breeding and genetics of forest trees. Proceedings of QFRI/CRC - SPF Symposium. Noosa, Australia. pp. 63-74

Barbour RC, Potts BM, Vaillancourt RE (2006) Gene flow between introduced and native Eucalyptus species: early-age selection limits invasive capacity of exotic $E$. ovata $\times E$. nitens $\mathrm{F} 1$ hybrids. For Ecol Manag 228:206-214

Barbour RC, Potts BM, Vaillancourt RE (2007) Gene flow between introduced and native Eucalyptus species: morphological analysis of tri-species and backcross hybrids involving E. nitens. Silv Genet 56:127-133

Barbour RC, Crawford AC, Henson M, Lee DJ, Potts BM, Shepherd M (2008) The risk of pollen-mediated gene flow from exotic Corymbia plantations into native Corymbia populations in Australia. For Ecol Manag 256:1-19

Barbour RC, Wise SL, McKinnon GE, Vaillancourt RE, Williamson GJ, Potts BM (2010) The potential for gene flow from exotic eucalypt plantations into Australia's rare native eucalypts. For Ecol Manag 260:2079-2087

Brawner JT, Dieters MJ, Nikles DG (2005) Mid-rotation performance of Pinus caribaea var. hondurensis hybrids with both $P$. oocarpa and $P$. tecunumanii: Hybrid superiority, stability of parental performance and potential for a multi-species synthetic breed. For Gen 12:1-13 
Costa e Silva J, Potts BM, Tilyard P (2012) Epistasis causes outbreeding depression in eucalypt hybrids. Tree Genet Genome $8: 249-265$

CPBR (2006) Euclid: Eucalypts of Australia. Centre for Plant Biodiversity Research. CDROM. CSIRO Publishing, Collingwood

Dickinson GR, Wallace HM, Lee DJ (2010) Controlled pollination methods for creating Corymbia hybrids. Silv Genet 59:233-241

Dickinson GR, Lee DJ, Wallace HM (2012) The influence of pre- and postzygotic barriers on hybridisation between Corymbia sections. Ann Bot 109:1215-1226

Ellis MF, Sedgley M, Gardner JA (1991) Interspecific pollen-pistil interaction in Eucalyptus L'Her. (Myrtaceae): the effect of taxonomic distance. Ann Bot 68:185-194

Field DL, Ayre DJ, Whelan RJ, Young AG (2009) Molecular and morphological evidence of natural interspecific hybridisation between the uncommon Eucalyptus aggregate and the widespread E. rubida and E. viminalis. Conserv Genet 10:881-896

Gore PL, Potts BM, Volker PW, Megalos J (1990) Unilateral crossincompatibility in Eucalyptus: the case of hybridisation between E. globulus and E. nitens. Aust J Bot 38:383-394

Griffin AR, Burgess IP, Wolf L (1988) Patterns of natural and manipulated hybridisation in the genus Eucalyptus L'Herit.-a review. Aust J Bot 36:41-66

Hill KD, Johnson LAS (1995) Systematic studies in the eucalypts 7. A revision of the bloodwoods, genus Corymbia (Myrtaceae). Telopea 6:185-504

Hung CD, Trueman SJ (2011) Topophysic effects differ between node and organogenic cultures of the eucalypt Corymbia torelliana $\times$ C. citriodora. Plant Cell Tissue Organ Cult 104:69-77

Iglesias-Trabado G, Wilstermann D (2008) Eucalyptus universalis, Global cultivated eucalypt forests map 2008. Version 1.0.1. GIT Forestry Consulting-Eucalyptologics. www.git-forestry.com, 1 August 2010

Lee DJ (2007) Achievements in forest tree genetic improvement in Australia and New Zealand. 2. Development of Corymbia species and hybrids for plantations in eastern Australia. Aust For 70:11-16

Lee DJ, Huth JR, Brawner JT, Dickinson GR (2009) Comparative performance of Corymbia hybrids and parental open-pollinated families in subtropical Queensland. Silv Genet 58:205-212

Lee DJ, Huth JR, Osborne DO, Hogg BW (2010) Selecting hardwood taxa for wood and fibre production in Queensland's subtropics. Aust For 73:106-114

Lewis T, Osborne D, Hogg B, Swift S, Ryan S, Taylor D, MacgregorSkinner J (2010) Tree growth relationships and silvicultural tools to assist stand management in private native spotted gum dominant forests in Queensland and northern New South Wales. Forest and Wood Products Australia, Report No. PNC075-0708

Lopez GA, Potts BM, Tilyard PA (2000) F1 hybrid inviability in Eucalyptus: the case of E. ovata $\times E$. globulus. Heredity $85: 242-250$

Nikles DG, Newton RS (1991) Correlations of breeding values in pure and hybrid populations of hoop pine and some southern pines in Queensland and relevance to breeding strategies. In: Dean CA (Ed), Proceedings of the $11^{\text {th }}$ Research Working Group 1 (Forest Genetics) Meeting, Mt Gambier, South Australia, pp 192-196

Parra-O C, Bayly MJ, Drinnan A, Udovicic F, Ladiges P (2009) Phylogeny, major clades and infrageneric classification of
Corymbia (Myrtaceae), based on nuclear ribosomal DNA and morphology. Aust Syst Bot 22:384-399

Potts BM, Dungey HS (2004) Interspecific hybridisation of Eucalyptus: key issues for breeders and geneticists. New Forest 27:115-138

Potts BM, Wiltshire RJE (1997) Eucalypt genetics and genecology. In: Williams JE, Woinarski JCZ (eds) Eucalypt ecology: individuals to ecosystems. Cambridge University Press, Cambridge, pp 5691

Potts BM, Volker PW, Tilyard PA, Joyce K (2000) The genetics of hybridisation in the temperate Eucalyptus. In: Dungey HS, Dieters MJ, Nikles DG (Eds) Hybrid breeding and genetics of forest trees. Proceedings of QFRI/CRC-SPF Symposium. Noosa, Australia. pp. 200-211

Potts BM, Barbour RC, Hingston AB, Vaillancourt RE (2003) Turner Review No. 6, Genetic pollution of native eucalypt gene poolsidentifying the risks. Aust J Bot 51:1-25

Pound LM, Wallwork MAB, Potts BM, Sedgley M (2003) Pollen tube growth and early ovule development following self- and crosspollination in Eucalyptus nitens. Sex Plant Reprod 16:59-69

Rix KD, Gracie AJ, Potts BM, Brown PH, Spurr CJ, Gore PL (2012) Paternal and maternal effects on the response of seed germination to high temperatures in Eucalyptus globulus. Ann For Sci. doi:10.1007/s13595-012-0190-7

Schierenbeck KA, Ellstrand NC (2009) Hybridisation and the evolution of invasiveness in plants and other organisms. Biol Invasions 11:1093-1105

Shepherd M, Pomroy P, Dieters M, Lee D (2007) Genetic control of propagation traits in a single $C$. torelliana $\times C$. variegata family. Can J For Res 37:2563-2574

Stanton BJ, Neale DB, Li S (2010) Populus breeding: from the classical to the genomic approach. In: Jansson S, Groover AT, Bhalerao RP (Eds) Plant genetics and genomics volume 8: genetics and genomics of Populus. Springer Science+Business Media, doi:10.1007/978-1-4419-1541-2 14. 309-348

Suitor S, Potts BM, Brown PH, Gracie AJ, Gore PL (2008) Postpollination capsule development in Eucalyptus globulus seed orchards. Aust J Bot 56:51-58

Vanden Broeck A, Villar M, Van Bockstaele E, Van Slycken J (2005) Natural hybridisation between cultivated poplars and their wild relatives: evidence and consequences for native poplar populations. Ann For Sci 62:601-613

Verma SK, Sharma VK (2011) Assessment of wood traits variation in the segregating populations of Eucalyptus hybrids. Indian Forester 137:732-738

Verma SK, Sharma VK, Bagchi SK (1999) The phenology of flowering of reciprocal F1 hybrids Eucalyptus citriodora Hook. $\times$ Eucalyptus torelliana F.v. Muell., $\mathrm{F}_{2}$ and $\mathrm{F}_{3}$ segregates and parent species at New Forest, Dehra Dun. Ann For 7:120-124

Volker PW, Potts BM, Borralho NMG (2008) Genetic parameters of intra- and inter-specific hybrids of Eucalyptus globulus and E. nitens. Tree Gen Genome 4:445-460

Wallwork MAB, Sedgley M (2005) Outcrossing in interspecific hybrids between Eucalyptus spathulata and E. platypus. Aust J Bot 53:347-355

Ziegenhagen B, Gneuss S, Rathmacher G, Leyer I, Bialozyt R, Heinze B, Liepelt S (2008) A fast and simple genetic survey reveals the spread of poplar hybrids at a natural Elbe river site. Conserv Genet 9:373-379 